\title{
Why are essential genes essential? - The essentiality of Saccharomyces genes
}

\author{
Zhaojie Zhang ${ }^{*}$ and Qun Ren \\ Department of Zoology and Physiology, University of Wyoming, Laramie, WY 82071, USA. \\ * Corresponding Author: Dr. Zhaojie Zhang, Department of Zoology and Physiology, University of Wyoming, Laramie, WY 82071, USA; \\ Tel: 307-766-3038; Email: zzhang@uwyo.edu
}

\begin{abstract}
Essential genes are defined as required for the survival of an organism or a cell. They are of particular interests, not only for their essential biological functions, but also in practical applications, such as identifying effective drug targets to pathogenic bacteria and fungi. The budding yeast Saccharomyces cerevisiae has approximately 6,000 open reading frames, 15 to $20 \%$ of which are deemed as essential. Some of the essential genes, however, appear to perform non-essential functions, such as aging and cell death, while many of the non-essential genes play critical roles in cell survival. In this paper, we reviewed and analyzed the levels of essentiality of the Saccharomyces cerevisiae genes and have grouped the genes into four categories: (1) Conditional essential: essential only under certain circumstances or growth conditions; (2) Essential: required for survival under optimal growth conditions; (3) Redundant essential: synthetic lethal due to redundant pathways or gene duplication; and (4) Absolute essential: the minimal genes required for maintaining a cellular life under a stress-free environment. The essential and nonessential functions of the essential genes were further analyzed.
\end{abstract}

doi: 10.15698/mic2015.08.218

Received originally: 10.04.2015;

in revised form: 23.05.2015,

Accepted 28.05.2015

Published 25.07.2015.

Keywords: cell death, cell survival, essential genes, growth conditions, Saccharomyces cerevisiae.
Abrreviations:
GO-Gene ontology,
$R O S$ - reactive oxygen species.

\section{DIFFERENT LEVELS OF ESSENTIALITY}

Generally speaking, essential genes are genes required for a cell or an organism to survive. Disruption or deletion of such genes causes cell death, indicating that these genes perform essential biological functions. Some of the essential genes, however, appear to have non-essential functions. FAS2, for example, encodes an $\alpha$-subunit of fatty acid synthetase, which catalyzes the synthesis of long-chain saturated fatty acids [1]. The null mutant could be rescued by fatty acid supplement. It is not clear why FAS2 functions as an essential gene. This also makes the term essential somewhat confusing, because essentiality depends on the environment in which cells live [2]. For example, a yeast gene required for respiration is essential only when ethanol or glycerol is the sole carbon source. Here, we have grouped the essential genes into four categories, depending on the circumstances or conditions these genes are required (Fig. 1).

(1) Conditional essential: these genes are essential only under certain circumstances or growth conditions. Auxotrophic genes are among the best examples for conditional essential. In the absence of uracil in culture medium, the orotidine-5'-phosphate decarboxylase gene, URA3, which catalyzes the sixth enzymatic step in the de novo biosyn- thesis of pyrimidines, becomes essential [3]. Many genes could be conditionally essential, but the "condition" is unknown (see below for discussion about PKC1). This could be one of the reasons why the molecular functions of more than $10 \%$ of the yeast protein-coding genes are still unknown. Many of these genes could be required or essential only under certain conditions, or stresses.

(2) Essential: refers to genes required for survival under the optimal growth condition. This is the most widely accepted definition of essential genes. The "optimal condition" though, is somewhat debatable. The yeast PKC1 gene, for example, encodes a protein serine/threonine kinase, which is essential for cell wall remodeling during growth [4]. Deletion of $P K C 1$ is lethal, but the lethality could be recovered by an osmotic stabilizer (such as sorbitol) [5], the "optimal growth condition" for this mutant. Another example is the Acetyl-CoA synthetase gene ACS2, which is required for fermentative growth on glucose. Null mutant is inviable in YPD medium, but is capable of respiratory growth on non-fermentable carbon sources, such as ethanol [6]. Therefore, the "optimal growth condition" for $\operatorname{acs} 2 \Delta$, is non-fermentable carbon sources, rather than glucose. 
(1)

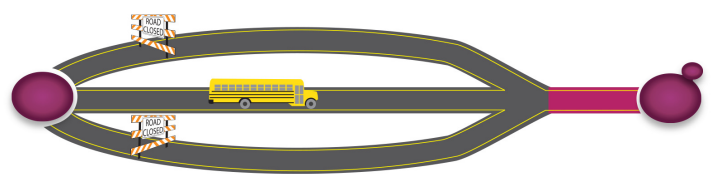

(2)

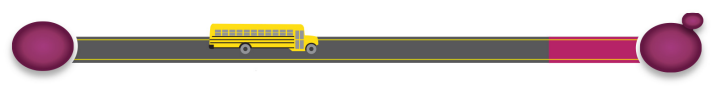

(3a)

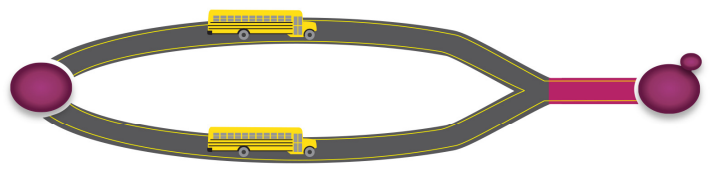

(3b)

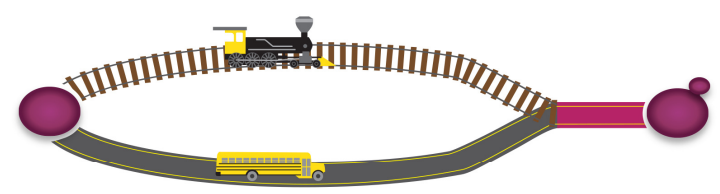

(3c)

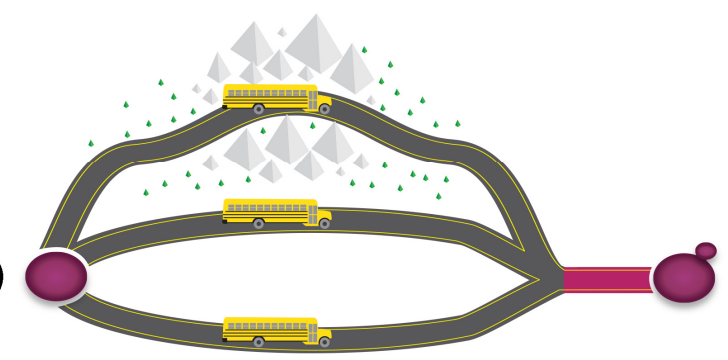

FIGURE 1: A symbolic illustration of different levels of essentiality of the Saccharomyces cerevisiae genes. (1) Conditional essential: when all other roads are closed, this alternative road becomes essential. (2) Essential: the only available road to your destination. (3a) Redundant essential: two duplicated/similar roads to your destination; (3b) Redundant essential: two different roads (pathways), but to the same destination; (3c) Redundant essential (synthetic sick): Two similar/different roads, plus a less preferred road, when the main roads become unavailable. The last part of the road (marked as red) represents the absolute essential.

The percentage of genes that are essential in the whole genome varies in different species, ranging from $\sim 2 \%$ in Drosophila melanogaster [7] to about $80 \%$ in Mycoplasma genitalium [8]. In the yeast Saccharomyces cerevisiae, about $20 \%$ of the whole genes are essential. The extremely low percentage of essential genes in Drosophila could be attributed to the methods (RNAi) used. RNAi is far less restrictive than the knock-outs used in $S$. cerevisiae and sometimes leads to hypomorphic results only [7]. Because of the importance of essential genes, yeast may have even evolved a protection mechanism to reduce the mutation rate of essential genes by placing them in the cold spots for mutation [9].

Most of the gene products, essential or not, form protein complexes to carry out their molecular functions [10]. A recent study showed that essential protein complexes tend to be formed by essential genes and nonessential complexes by nonessential genes, suggesting the existence of modular essentiality [11].
There are also cases where an essential gene becomes non-essential by deletion of another (non-essential) gene. FtsZ is a tubulin-like gene in bacteria that forms the cytoskeletal framework of the cytokinetic ring. It is essential for cell division in wild type bacteria. However, in the L-form of Bacillus subtilis, caused by a single amino acid mutation of yqiG gene, Fts $Z$ becomes non-essential. The $B$. subtilis cells proliferate by extrusion and resolution, instead of binary fission [12].

(3) Redundant essential (synthetic lethal/sick): eukaryotic cells evolve multiple redundant pathways to resist environmental and genetic perturbations [13]. One way of producing a redundant pathway is the so called whole genome duplication, a product of nondisjunction during meiosis, which results in additional copies of the entire genome [14]. Gene duplication may also provide stability to an organism by buffering the effect of harmful mutations [15]. The Saccharomyces genome contains $\sim 1,060$, or $\sim 530$ pairs of paralogs, homologous sequences evolved from gene duplication. Synthetic lethality occurs when mutations of both genes cause cell death, while single deletion of either gene is viable. The paralogous genes BDF1 and $B D F 2$, for example, encode bromodomain-containing transcription factors [16]. Double deletion is lethal, while single deletion of either BDF1 or BDF2 has no apparent phenotype [17]. In addition, $b d f 1 \Delta$ is sensitive to salt stress and the sensitivity can be suppressed by over-expression of $B D F 2$ [18]. Not all paralogous genes, however, are synthetic lethal. The paralogs $C Y C 1$ and $C Y C 7$ encode two isoforms of cytochrome c, iso-1-cytochrome $c$ and iso-2-cytochrome c, respectively. Iso-1-cytochrome c and iso-2-cytochrome c constitute approximately $95 \%$ and $5 \%$ of the total amount of cytochrome c in aerobically grown cells [19]. Double deletion of CYC1 and CYC7 completely blocks growth on non-fermentable carbon sources, but the growth is normal on rich glucose medium [20]. As for essentiality, $B D F 1 / B D F 2$ should be considered as redundant essential, while $C Y C 1 / C Y C 7$ should be considered as conditional essential. Another example of conditional essential is PET9, which encodes the major ADP/ATP translocator of the mitochondrial inner membrane [21]. PET9 has two paralogs, $A A C 1$ and $A A C 3$, both are expressed at a lower level compared to PET9. Similar to $C Y C 1 / C Y C 7$ double deletion, the triple deletion of $P E T 9 / A A C 1 / A A C 3$ is inviable only under anaerobic conditions [22]. A study in Caenorhabditis elegans showed that the non-essential (conditional essential) genes are actually duplicated more often, while essential genes (become redundant essential after duplication) are less often duplicated [15]. It is unclear how many of the 530 pairs of paralogs in yeast were evolved from nonessential (conditional essential), or essential genes.

(4) Absolute essential (minimal genes): genes that are necessary and sufficient for supporting cellular life in an environment that provides all necessary nutrients and is free from any stress [23]. These genes are often referred as minimal genes, and collectively as the minimal gene set. The "stress-free" environment, although easily defined, is difficult to implement in practice, since it is close to impossible to create such a "stress-free" environment. 
The parasitic bacterium Mycoplasma genitalium has an extremely small genome of $\sim 580 \mathrm{~kb}$, containing only 482 protein-coding genes. It offers one of the best organisms to study minimal genes [24]. Among the 482 genes, 382 were identified as essential and 256 were suggested as minimal genes [25]. These minimal genes are mainly involved in DNA replication, protein translation and metabolism. A set of genes required for recombination and DNA repair was also identified in the minimal genes [25], suggesting that DNA repair is a fundamental process required for cell survival.

\section{THE ESSENTIAL FUNCTIONS OF THE ESSENTIAL GENES}

To survive, an organism must be able to perform at least two fundamental functions: obtain energy, and reproduce. Cells must convert nutrients from foods, such as proteins, fats and sugars, into adenosine triphosphate (ATP), the ultimate source of energy for living cells. This process of energy conversion is referred as metabolism. Gene ontology (GO) analysis [26] of the 1120 essential genes of Saccharomyces cerevisiae [27] indicates that about $74 \%$ of the essential genes are involved in metabolic process and at least $14 \%$ in cell cycle regulation, the two essential functions for cell survival. To keep its simplicity, the discussion below only refers to these essential genes, unless noted otherwise. It should be noted that the genome-wide deletion set for Saccharomyces [27] also contains multiple mutations that can affect phenotypes.

\section{Essential genes and metabolism}

More than 700 essential genes are involved in this process that is essential for converting nutrients and providing energy for cells. It includes both catabolism (about 120 genes) and biosynthesis (about 450 genes) of nucleic acids, proteins, carbohydrate, lipids and other macromolecules. Among the $\sim 120$ catabolic related genes, 17 are involved in autophagy, a process for macromolecule recycling and intracellular structure remodeling. Recent studies have shown that autophagy plays a much greater physiological role, such as response to starvation and other stress conditions [28-29]. In addition to the 17 essential genes, there are about 70 non-essential genes (including conditional essential and redundant essential, such as ATG33 and SCM4) involved in autophagy.

\section{Essential genes and cell division/reproduction}

Reproduction is one of the fundamental functions for cell survival. Among the 158 (14\%) cell cycle related essential genes, the most important processes of these genes involved include: 1) Mitotic cell cycle, 2) Cytoskeleton organization, and 3) Response to stress and stimulus.

1) Mitotic cell cycle: Not surprisingly, more than half of the essential genes in mitosis are involved in nuclear division, including DNA replication, DNA repair, spindle assembly checkpoint, chromosome condensation and chromosome segregation. Yeast can reproduce either via asexual reproduction (mitosis) or sexual reproduction (meiosis). Meiosis, therefore, is not an essential function for cells to survive. It was puzzling to see 39 out of the 158 cell cyclerelated essential genes regulate meiosis. Further analysis revealed that none of the 39 genes are meiotic specific. They are involved in both mitosis and meiosis. The cohesin maintenance factor gene PDS5, for example, is required for sister chromatid condensation and cohesion during mitosis [30-31]. It is also required for sister chromatid cohesion in meiosis [32]. Some genes, such as DSN1, which encodes an essential component of the MIND kinetochore complex [33], are essential for meiosis [34], but their essentiality for survival is more likely due to their functions in mitosis.

2) Cytoskeleton organization: Saccharomyces cerevisiae reproduces through budding, a stereotypical pattern of polarized growth. Cytoskeleton organization plays an essential role in all steps of cell growth and cell division. The microtubule cytoskeleton related proteins (such as IPL1, and SPC105) are mostly required for nuclear division, including spindle checkpoints. The actin cytoskeleton related proteins are involved in organelle localization, vesicle transport and cytokinesis, such as the septin ring organization. The actin cytoskeleton of budding yeast consists of both the actin cortical patch and the actin cables. The actin cortical patches show a polarized distribution while the actin cables are oriented along the mother-bud axis during cell division [35].

3) Response to stress or stimulus: Faithful DNA replication and transmission of hereditary information is critical to cell survival. Not surprisingly, the majority of these essential stress-response genes are involved in genome integrity checkpoints, DNA damage and DNA repair. Rad53, for example, is a DNA damage response protein. It is required for cell cycle checkpoint function in response to DNA damage [36].

\section{THE "NON-ESSENTIAL" FUNCTIONS OF THE ESSENTIAL GENES}

\section{Essential genes and cell death}

Cell death, as opposed to cell survival, is not an essential function. GO analysis, however, revealed that at least five essential genes, BIR1, MCD1, ESP1, PDS5 and CDC48 are involved in cell death in budding yeast. Cautions should be taken when using the GO analysis. While it works well on large scales, it may provide inaccurate information on specific genes. PET9 for example, is listed as one of the essential genes involved in cell death. PET9 is required for mitochondrial outer membrane permeabilization and cytochrome c release [37], it is a conditional essential, rather than an essential gene.

Interestingly, all five cell death-related genes play critical roles in mitotic nuclear division. Induction of apoptotic cell death is likely a result of their "secondary" functions or simply by loss of function. Bir1, for example, is a homolog of human survivin and a subunit of the chromosomal passenger complex, which regulates chromosome segregation [38-39]. Under oxidative stress, however, the yeast Bir1 becomes a substrate for Nma111, the homologue of the human pro-apoptotic serine protease Omi/HtrA2BIR1, which mediates apoptotic cell death [40]. Deletion of BIR1 


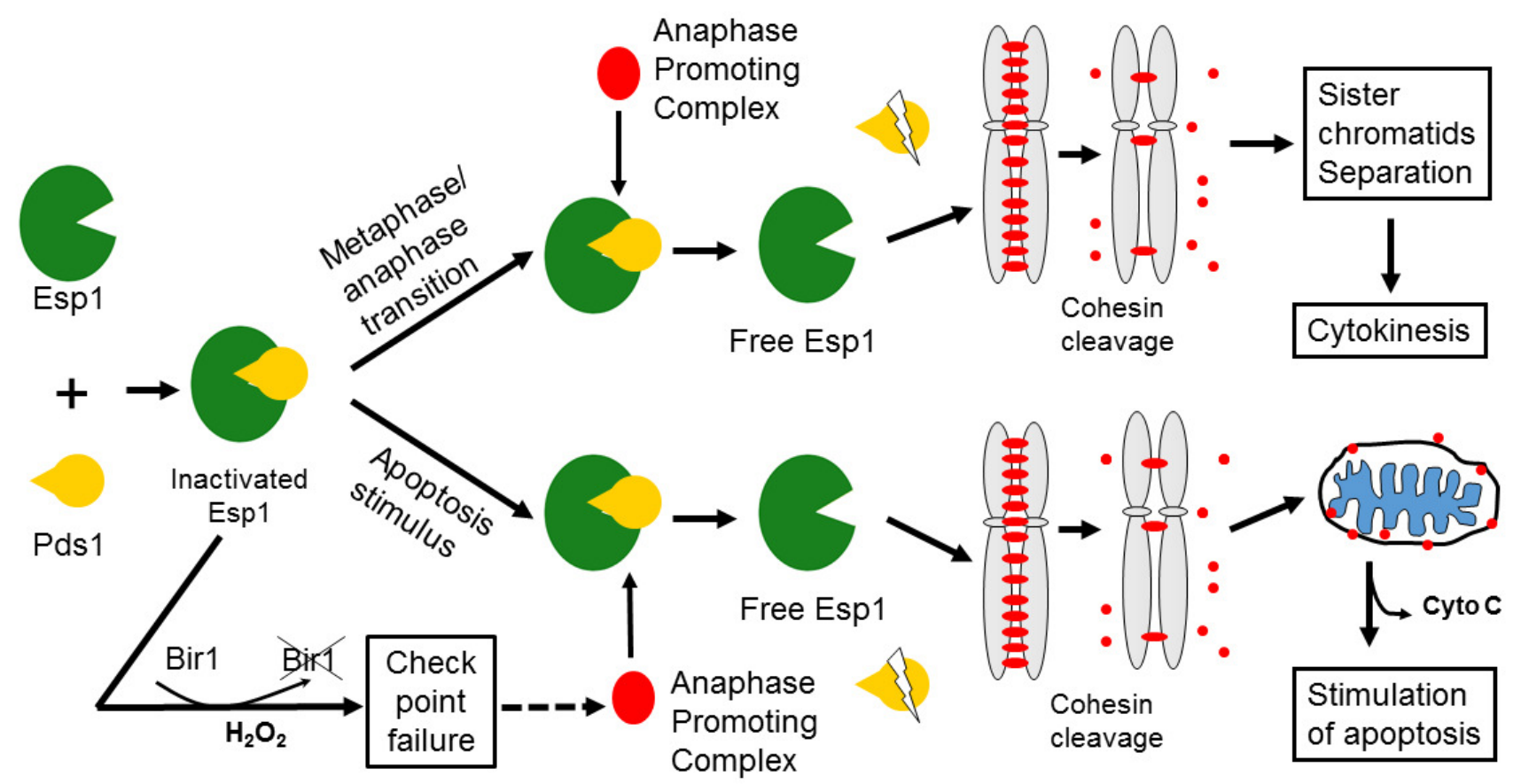

FIGURE 2: Involvement of essential genes (ESP1, MCD1 and BIR1) in apoptotic cell death. Modified according to [43].

also causes malfunction of the spindle assembly checkpoint and apoptosis [41], suggesting a possible connection between the functions in checkpoint regulation and antiapoptosis.

The yeast Esp1 is a caspase-like cysteine protease [42]. During metaphase-anaphase transition, Esp1 is released by its inhibitor Pds1, triggering sister chromatid separation by cleavage of the cohesin protein Mcd1. Upon oxidative stress, the Esp1 is prematurely released and cleaves Mcd1. The C-terminal fragment of Mcd1 is translocated into mitochondria and triggers the release of cytochrome $c$ and consequently apoptotic cell death (Fig. 2) [43], suggesting that Mcd1 actively participates in cell death. It would be interesting to see if any other essential proteins perform similar functions, i.e., triggering cell death under stress conditions, to ensure their essentiality.

Because of the nature of the essential genes - cells die without them, a still unanswered question is how cells die in the absence of an essential gene. Studies indicate that mutants of yeast essential genes could die apoptotically. In fact, the very first paper published on yeast apoptosis by Frank Madeo and his colleagues involved CDC48, an essential gene that regulates mitotic spindle disassembly. In this pioneer study, they reported that mutants in CDC48 show typical markers of apoptosis [44]. Mutation of another essential gene, PDS5, was also reported to lead to features of apoptosis (Fig. 3) [45-46]. What is unclear is whether the apoptotic cell death caused by mutation of CDC48 or PDS5 is due to the loss of functions of the essential gene, or like $M C D 1$, the essential gene actively participates in the cell death. If it is due to the loss of function, what are the triggers or inducers of the cell death?

In addition to cell death, GO analysis revealed that five essential genes are related to aging, another "nonessential" function for cell survival. Similar to genes in cell death, all these five essential genes (ACT1, DNA2, NMT1, $C D C 25$ and $P G A 3)$ are involved in other essential functions and defects could lead to accumulation of damage that leads to aging. ACT1 for example, encodes the essential gene for actin, a cytoskeleton protein critical for many cellular processes, such as polarized cell growth [47]. It has been shown that enhanced actin dynamics can increase the yeast lifespan, while decrease of actin dynamics causes depolarization of the mitochondrial membrane, production of reactive oxygen species (ROS) and cell death [48]. Mutation of $C D C 25$, on the other hand, enhances the lifespan in both yeast [49] and mice [50]. CDC25 encodes a guanine nucleotide exchange factor, which is required for glucose metabolism by activing the Ras GTPases [51]. Mutation of $C D C 25$ reduces glucose/calorie consumption and possibly reduces the level of ROS [49].

\section{Essential genes and mitochondrial organization}

Mitochondria are organelles present in virtually all eukaryotic cells. They are the site for numerous important cellular metabolic processes. Although S. cerevisiae is able to carry out fermentative growth on carbon sources such as glucose in the absence of mitochondrial DNA, maintenance of the mitochondrial compartment is essential for survival. 


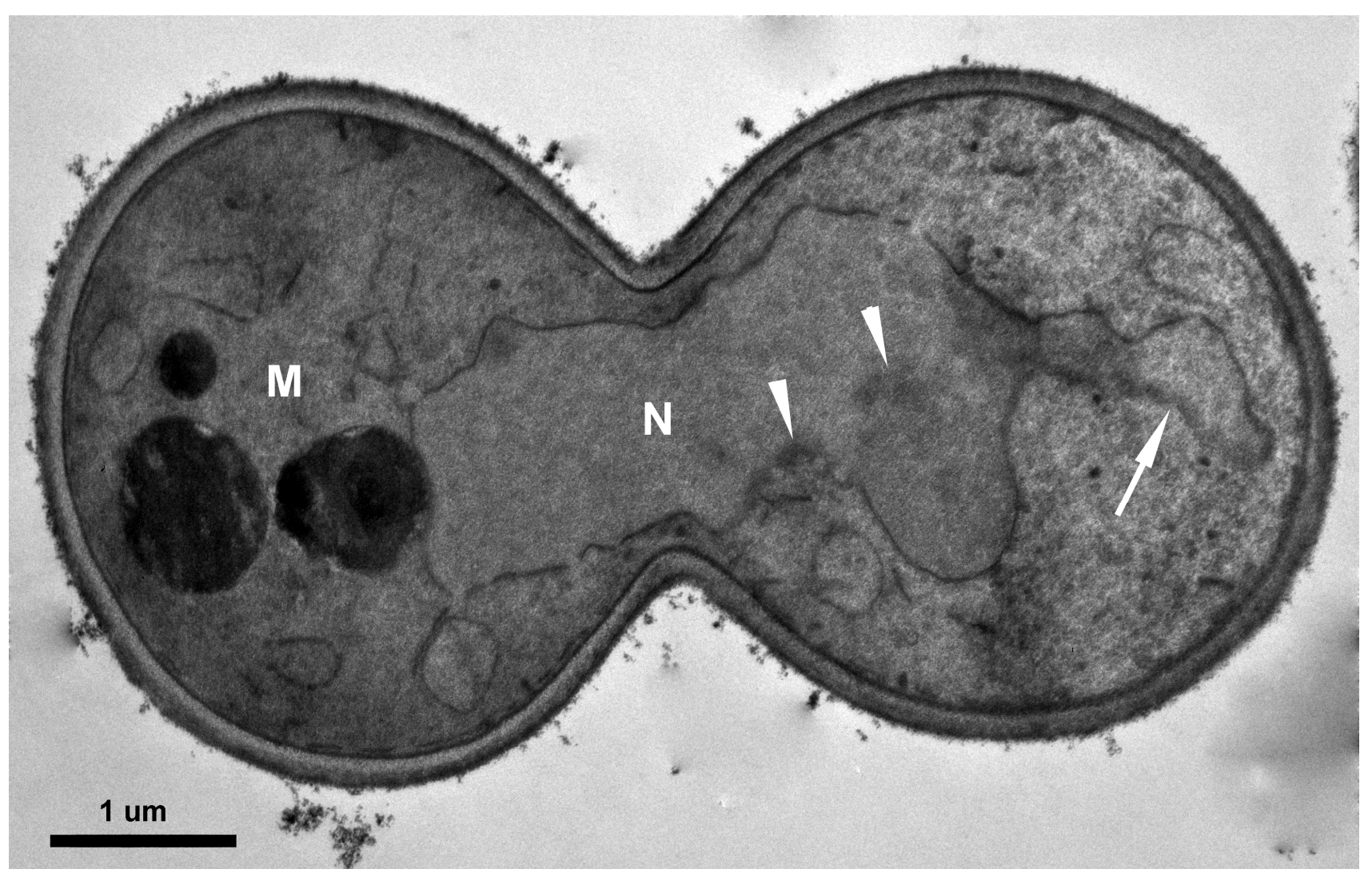

FIGURE 3: TEM image of pds5-1 mutant showing the nucleus unable to divide when switched to non-permissive temperature. It also shows chromatin condensation (arrowhead) and nucleus with protruding tube (arrow), both typical markers of apoptotic cell death. $\mathrm{N}=$ nucleus. $\mathrm{M}=$ mother cell, as suggested by the vacuole.

GO analysis revealed that about 50 essential genes are involved in mitochondrial organization. A systematic large screening of the 768 essential genes with a regulatable promoter identified about 120 genes that are essential for mitochondrial morphology [52]. The majority of these genes are involved in protein targeting/import/transport to mitochondria. Most prominently are the TOM (translocase of outer membrane) proteins (TOM20, TOM22 and TOM40), and the TIM (translocase of inner membrane) proteins (TIM10, TIM12, TIM17, TIM22, TIM23, TIM44, TIM50 and TIM54). These are essential proteins of either the outer or inner mitochondrial membrane that are important for import/insertion of proteins into the mitochondrial inner membrane and to the mitochondrial matrix.

Besides producing ATP as the energy source, mitochondria are involved in a number of essential biosynthetic pathways, such as the ergosterol biosynthetic pathway (encoded by ERG29), which is essential to yeast viability [53]. Mitochondria also play a central function in the biogenesis of cellular iron-sulphur proteins, such as Rli1, which is required for ribosome biogenesis and protein translation [54]. Recent studies also demonstrated that mitochondria are key regulators of apoptotic cell death [55-57].

\section{CONCLUSIONS AND PERSPECTIVES}

The reason essential genes are essential is that these genes carry out biological functions that are necessary for cell survival. Many of the "non-essential genes" also perform essential functions. They are deemed non-essential, not because of their functions, but rather their functions are backed up with redundant genes or pathways. Therefore, the definition of essential genes should be expanded to include all genes that carry out essential functions, rather than simply required for survival. The difficulty of using this term is that a lot of essential biological functions are yet to be uncovered, a challenge and a future direction towards a better understanding of cell biology. Another challenge is to decipher the essential function(s) each gene carries. The problem for the "non-essential", or conditionally essential genes, is to identify the unknown conditions/circumstances under which the genes are required, because mutants deleted for these genes lack any obvious phenotype under normal conditions. The challenge for essential genes is that death is the only "phenotype" in the absence of any essential gene. The generation of the tetracycline-regulatable alleles of essential genes [58] greatly facilitates large scale studies on essential genes, such as those involved in secretion [59], mitochondria [52] or G-protein signaling [60]. 
These studies often identify the non-essential, or secondary functions of the essential genes, which may help further identify the essential functions. The essential gene YPP1/YGR198W was first discovered as a gene required for protection from $\alpha$-synuclein, a protein that causes Parkinson's disease in human [61]. It is also shown that YPP1 interacts with genes involved in protein sorting and secretion, which further led the discovery of its primary essential functions [62-63]. In addition to discovery of the essential functions, it is important to understand their involvement in cell death. Do all the essential genes actively participate in cell death? Is there a universal trigger of cell death in the absence of an essential gene?

\section{ACKNOWLEDGMENTS}

This study was supported in part by an Institutional Development Award (IDeA) from the National Institute of General Medical Sciences of the National Institutes of Health under grant number P30 GM103398. The authors thank Ms. Cassidy

\section{REFERENCES}

1. Fichtlscherer F, Wellein C, Mittag M, Schweizer E (2000). A novel function of yeast fatty acid synthase. Subunit alpha is capable of selfpantetheinylation. Eur J Biochem 267:2666-71.

2. D'Elia MA, Pereira MP, Brown ED (2009). Are essential genes really essential? Trends Microbiol 17:433-8.

3. Umezu K, Amaya T, Yoshimoto A, Tomita K (1971). Purification and properties of orotidine-5'-phosphate pyrophosphorylase and orotidine-5'-phosphate decarboxylase from baker's yeast. J Biochem 70:249-62.

4. Lee KS, Irie K, Gotoh Y, Watanabe $Y$, Araki H, Nishida E, Matsumoto $K$, Levin DE (1993). A yeast mitogen-activated protein kinase homolog (Mpk1p) mediates signaling by protein kinase C. Mol Cell Biol 13:3067-75.

5. Sussman A, Huss K, Chio LC, Heidler S, Shaw M, Ma D, Zhu G, Campbell RM, Park TS, Kulanthaivel P, Scott JE, Carpenter JW, Strege MA, Belvo MD, Swartling JR, Fischl A, Yeh WK, Shih C, Ye XS (2004). Discovery of cercosporamide, a known antifungal natural product, as a selective Pkc1 kinase inhibitor through high-throughput screening. Eukaryot Cell 3:932-43.

6. Van den Berg MA, Steensma HY (1995). ACS2, a Saccharomyces cerevisiae gene encoding acetyl-coenzyme A synthetase, essential for growth on glucose. Eur J Biochem 231:704-13.

7. Boutros M, Kiger AA, Armknecht S, Kerr K, Hild M, Koch B, Haas SA, Paro R, Perrimon N; Heidelberg Fly Array Consortium (2004). Genomewide RNAi analysis of growth and viability in Drosophila cells. Science 303:832-5.

8. Chen WH, Minguez P, Lercher MJ, Bork P (2012). OGEE: an online gene essentiality database. Nucleic Acids Res 40 (Database issue):D901-6.

9. Rattray A, Santoyo G, Shafer B, Strathern JN (2015). Elevated mutation rate during meiosis in Saccharomyces cerevisiae. PLoS Genet 11:e1004910.

10. Hartwell LH, Hopfield JJ, Leibler S, Murray AW (1999). From molecular to modular cell biology. Nature 402:C47-C52.

11. Ryan CJ, Krogan NJ, Cunningham P, Cagney G (2013). All or nothing: protein complexes flip essentiality between distantly related eukaryotes. Genome Biol Evol 5:1049-59.
Mittelstadt (University of Wyoming Graphic design services) for her help designing the illustration (Figure 1).

\section{CONFLICT OF INTEREST}

The authors declare no conflict of interest.

\section{COPYRIGHT}

(C) 2015 Zhang and Ren. This is an open-access article released under the terms of the Creative Commons Attribution (CC BY) license, which allows the unrestricted use, distribution, and reproduction in any medium, provided the original author and source are acknowledged.

Please cite this article as: Zhaojie Zhang and Qun Ren (2015). Why are essential genes essential? - The essentiality of Saccharomyces genes. Microbial Cell 2(8): 280-287. doi: 10.15698/mic2015.08.218

12. Leaver M, Domínguez-Cuevas $P$, Coxhead JM, Daniel RA, Errington $\mathrm{J}$ (2009). Life without a wall or division machine in Bacillus subtilis. Nature 457:849-53.

13. Hartwell, L (2004). Genetics. Robust interactions. Science 303, 774-775.

14. Kellis M, Birren BW, Lander ES (2004). Proof and evolutionary analysis of ancient genome duplication in the yeast Saccharomyces cerevisiae. Nature 428:617-24.

15. Woods S, Coghlan A, Rivers D, Warnecke T, Jeffries SJ, Kwon T, Rogers A, Hurst LD, Ahringer J (2013). Duplication and retention biases of essential and non-essential genes revealed by systematic knockdown analyses. PLoS Genet (5):e1003330.

16. Lygerou $Z$, Conesa $C$, Lesage $P$, Swanson RN, Ruet A, Carlson $M$, Sentenac A, Séraphin B (1994). The yeast BDF1 gene encodes a transcription factor involved in the expression of a broad class of genes including snRNAs. Nucleic Acids Res 22:5332-40.

17. Sawa C, Nedea E, Krogan N, Wada T, Handa H, Greenblatt J and Buratowski S (2004). Bromodomain factor 1 (Bdf1) is phosphorylated by protein kinase CK2. Mol Cell Biol 24:4734-4742.

18. Fu J, Hou J, Liu L, Chen L, Wang M, Shen Y, Zhang Z, Bao X (2013). Interplay between BDF1 and BDF2 and their roles in regulating the yeast salt stress response. FEBS J 280:1991-2001.

19. Downie JA, Stewart JW, Brockman N, Schweingruber AM and Sherman F (1977). Structural Gene for Yeast Iso-2-cytochrome c J. Mol Biol 113:369-384.

20. Barrientos A, Pierre D, Lee J, Tzagoloff A (2003). Cytochrome oxidase assembly does not require catalytically active cytochrome C. J Biol Chem 278:8881-7.

21. Lawson JE1, Douglas MG (1988). Separate genes encode functionally equivalent ADP/ATP carrier proteins in Saccharomyces cerevisiae. Isolation and analysis of AAC2. J Biol Chem 263:14812-8.

22. Drgon T, Sabová L, Nelson N, Kolarov J (1991). ADP/ATP translocator is essential only for anaerobic growth of yeast Saccharomyces cerevisiae. FEBS Lett 289:159-62.

23. Koonin EV (2000). How many genes can make a cell: the minimalgene-set concept. Annu Rev Genomics Hum Genet 1: 99-116. 
24. Glass JI, Assad-Garcia N, Alperovich N, Yooseph S, Lewis MR, Maruf $M$, Hutchison CA III, Smith HO, Venter JC (2006). Essential genes of a minimal bacterium. Proc Natl Acad Sci U S A 103:425-30.

25. Mushegian AR, Koonin EV (1996). A minimal gene set for cellular life derived by comparison of complete bacterial genomes. Proc Natl Acad Sci U S A 93:10268-10273.

26. GO analysis is based on the analysis tools at Saccharomyces Genome Database including Go Term Finder (http://www.yeastgenome.org/cgi-bin/GO/goTermFinder.pl) and Go Slim Mapper (http://www.yeastgenome.org/cgibin/GO/goSlimMapper.pl).

27. The essential gene list is based on the Yeast Deletion Project (yeastdeletion.stanford.edu).

28. Madeo F, Zimmermann A, Maiuri MC, Kroemer G (2015). Essential role for autophagy in life span extension. J Clin Invest 125:85-93.

29. Mizushima N (2007). Autophagy: process and function. Genes Dev 21:2861-73.

30. Hartman T, Stead K, Koshland D, Guacci V (2000). Pds5p is an essential chromosomal protein required for both sister chromatid cohesion and condensation in Saccharomyces cerevisiae. J Cell Biol 151:613-26

31. Panizza S, Tanaka T, Hochwagen A, Eisenhaber F, Nasmyth K (2000). Pds5 cooperates with cohesin in maintaining sister chromatid cohesion. Curr Biol 10:1557-64.

32. Zhang Z, Ren Q, Yang H, Conrad MN, Guacci V, Kateneva A, Dresser ME (2005). Budding yeast PDS5 plays an important role in meiosis and is required for sister chromatid cohesion. Mol Microbiol 56:670-80.

33. Euskirchen GM (2002). Nnf1p, Dsn1p, Mtw1p, and Nsl1p: a new group of proteins important for chromosome segregation in Saccharomyces cerevisiae. Eukaryot Cell 1:229-40.

34. Sarkar S, Shenoy RT, Dalgaard JZ, Newnham L, Hoffmann E, Millar $J B$, Arumugam $P$ (2013). Monopolin subunit Csm1 associates with MIND complex to establish monopolar attachment of sister kinetochores at meiosis I. PLoS Genet 9:e1003610.

35. Adams A, Pringle J (1984). Relationship of actin and tubulin distribution to bud growth in wild-type and morphogenetic-mutant Saccharomyces cerevisiae. J Cell Biol 98:934-945.

36. Weinert TA, Kiser GL, Hartwell LH (1994). Mitotic checkpoint genes in budding yeast and the dependence of mitosis on DNA replication and repair. Genes Dev 8:652-65.

37. Pereira C, Camougrand N, Manon S, Sousa MJ, Côrte-Real M (2007). ADP/ATP carrier is required for mitochondrial outer membrane permeabilization and cytochrome $c$ release in yeast apoptosis. Mol Microbiol 66:571-82.

38. Uren AG, Beilharz T, O'Connell MJ, Bugg SJ, van Driel R, Vaux DL, Lithgow T (1999). Role for yeast inhibitor of apoptosis (IAP)-like proteins in cell division. Proc Natl Acad Sci U S A 96:10170-75.

39. Yoon HJ and Carbon J (1999). Participation of Bir1p, a member of the inhibitor of apoptosis family, in yeast chromosome segregation events. Proc Natl Acad Sci USA 96: 13208-13213.

40. Walter D, Wissing S, Madeo F, Fahrenkrog B (2006). The inhibitorof-apoptosis protein Bir1p protects against apoptosis in S. cerevisiae and is a substrate for the yeast homologue of Omi/HtrA2. J Cell Sci 119(Pt 9):1843-51.

41. Ren Q, Liou LC, Gao Q, Bao X, Zhang Z (2012). Bir1 deletion causes malfunction of the spindle assembly checkpoint and apoptosis in yeast. Front Oncol 2:93.
42. Uhlmann F, Wernic D, Poupart MA, Koonin EV, Nasmyth K (2000). Cleavage of cohesin by the CD clan protease separin triggers anaphase in yeast. Cell 103:375-86.

43. Yang $H$, Ren $Q$, Zhang Z (2008). Cleavage of Mcd1 by caspase-like protease Esp1 promotes apoptosis in budding yeast. Mol Biol Cell 19:2127-34

44. Madeo F, Fröhlich E, Fröhlich KU (1997). A yeast mutant showing diagnostic markers of early and late apoptosis. J Cell Biol 139:729-34.

45. Ren $Q$, Yang $H$, Rosinski $M$, Conrad MN, Dresser ME, Guacci V, Zhang $Z$ (2005). Mutation of the cohesin related gene PDS5 causes cell death with predominant apoptotic features in Saccharomyces cerevisiae during early meiosis. Mutat Res 570:163-73.

46. Ren Q, Yang H, Gao B and Zhang Z (2008). Global transcriptional analysis of yeast cell death induced by mutation of sister chromatid cohesin. Comparative and Functional Genomics ID 634283.

47. Botstein D, Amberg D, Molholland J, Huffaker T, Adams A, Drubin D, Stearns T (1997). "The yeast cytoskeleton." Pp. 1-90 in The Molecular and Cellular Biology of the Yeast Saccharomyces: Cell Cycle and Cell Biology, edited by Pringle JR, Broach JR and Jones EW. Cold Spring Harbor, NY: Cold Spring Harbor Laboratory Press

48. Gourlay CW, Carpp LN, Timpson P, Winder SJ, Ayscough KR (2004). A role for the actin cytoskeleton in cell death and aging in yeast. J Cell Biol 164:803-9.

49. Lin SJ, Defossez PA, Guarente L (2000). Requirement of NAD and SIR2 for life-span extension by calorie restriction in Saccharomyces cerevisiae. Science 289:2126-8.

50. Borrás C, Monleón D, López-Grueso R, Gambini J, Orlando L, Pallardó FV, Santos E, Viña J, Font de Mora J (2011). RasGrf1 deficiency delays aging in mice. Aging (Albany NY). 3:262-76

51. Jones S, Vignais ML, Broach JR (1991). The CDC25 protein of Saccharomyces cerevisiae promotes exchange of guanine nucleotides bound to ras. Mol Cell Biol 11:2641-6.

52. Altmann K and Westermann B (2005). Role of Essential Genes in Mitochondrial Morphogenesis in Saccharomyces cerevisiae. Mol Biol Cell. 16:5410-5417.

53. Moretti-Almeida G, Netto LE, Monteiro G (2013). The essential gene YMR134W from Saccharomyces cerevisiae is important for appropriate mitochondrial iron utilization and the ergosterol biosynthetic pathway. FEBS Lett 587:3008-13.

54. Kispal G, Sipos K, Lange H, Fekete Z, Bedekovics T, Janáky T, Bassler J, Aguilar Netz DJ, Balk J, Rotte C, Lill R (2005). Biogenesis of cytosolic ribosomes requires the essential iron-sulphur protein Rli1p and mitochondria. EMBO J 24:589-98.

55. Newmeyer DD, Ferguson-Miller S (2003). Mitochondria: releasing power for life and unleashing the machineries of death. Cell 112:48190.

56. McBride HM, Neuspiel M, Wasiak S (2006). Mitochondria: more than just a powerhouse. Curr Biol 16:R551-60.

57. Eisenberg T, Büttner S, Kroemer G, Madeo F (2007). The mitochondrial pathway in yeast apoptosis. Apoptosis 12:1011-23.

58. Gari E, Piedrafita L, Aldea M, Herrero E (1997). A set of vectors with a tetracycline-regulatable promoter system for modulated gene expression in Saccharomyces cerevisiae. Yeast 13:837-848.

59. Davydenko SG, Juselius JK, Munder T, Bogengruber E, Jäntti J, Keränen $S$ (2004). Screening for novel essential genes of Saccharomyces cerevisiae involved in protein secretion. Yeast 21:46371. 
60. Cappell SD, Baker R, Skowyra D, Dohlman HG (2010). Systematic analysis of essential genes reveals important regulators of $\mathrm{G}$ protein signaling. Mol Cell 38:746-57.

61. Flower TR1, Clark-Dixon C, Metoyer C, Yang H, Shi R, Zhang Z, Witt SN (2007). YGR198w (YPP1) targets A30P alpha-synuclein to the vacuole for degradation. J Cell Biol 177:1091-104.
62. Baird D, Stefan C, Audhya A, Weys S, Emr SD (2008). Assembly of the PtdIns 4-kinase Stt4 complex at the plasma membrane requires Ypp1 and Efr3. J Cell Biol 183:1061-74.

63. Zhai C, Li K, Markaki V, Phelan JP, Bowers K, Cooke FT, Panaretou B (2008). Ypp1/YGR198w plays an essential role in phosphoinositide signaling at the plasma membrane. Biochem J 415:455-66. 